such as the US plan to visit the Moon and Mars. It would have been more appropriate to question the place of science and basic research in our society. This has to be carefully argued to convince a broad audience, but I am afraid he is preaching to the converted.
The main aim of the book is to describe what astronomers have learned so far about the first stars and galaxies in the early Universe, but it does much more than this. Given the huge strides being made in exploring the Universe, physicists are having to find new and different ways of explaining what they see. This creates the feeling that we have reached the end of the beginning and are looking ahead.

Patrick Petitjean is at the Institut d'Astrophysique de Paris, 98 bis Boulevard Arago, 75014 Paris, France.

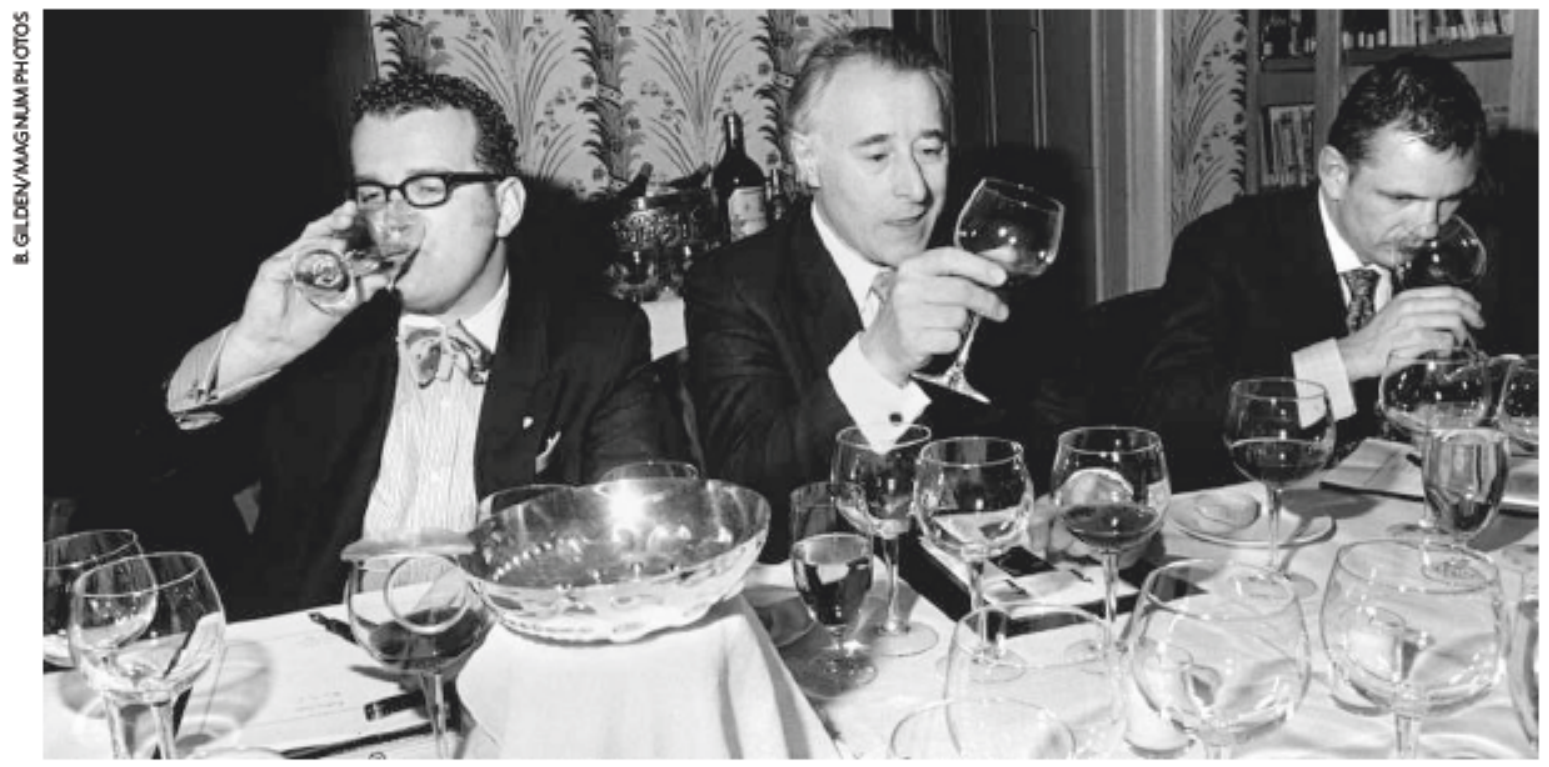

Glass distinction:it's all in the flavour, the colour and the bouquet for those who wish to enjoy wines or identify them in a 'blind' tasting.

\section{A question of taste}

Molecular Gastronomy: Exploring the Science of Flavor

by Hervé This (transl. by M. B. DeBevoise) Columbia University Press: 2006. 360 pp. $\$ 29.95$

\section{John Piggott}

In the introduction to this book, Herve This says what he means by 'molecular gastronomy', explains its origins and carefully differentiates it from food science. In essence, he defines molecular gastronomy as the science (physics, chemistry and biology) of enjoying food. However, insofar as it is science applied to food, it is simplest to regard it as a branch of food science, concerned particularly, although not exclusively, with the transformation of ingredients to foods and their subsequent consumption. He applies it especially to culinary, rather than industrial, processes.

Molecular Gastronomy is an anthology of columns from Pour la Science (the French edition of Scientific American), mostly published in the 1990s. The short chapters are organized into four sections. The first part describes investigations and explanations of traditional cooking methods, and the second covers the physiology of flavour (mostly taste). The third and longest part is composed largely of discussions of journal articles and news stories, with most being explanations of how traditional products work. Finally, applications of recent science and technology are discussed to suggest new products derived from existing ones. At the end there is a glossary, a list of further reading and a good index.

One or two of the explanations in the first section are not convincing but would make a good starting point for anyone wanting to take them further. Elsewhere the descriptions and explanations of recent science are clear and coherent but uncritical. For example, he cites a statistical analysis of some Scotch whisky tasting data that demonstrates only that if you use inappropriate data in a statistical analysis you get meaningless results. There seems to be disproportionate attention paid to alcoholic beverages, including a chapter about warming and chilling wine. This did not go far enough for me; there is no help with practical problems, such as how long you should you leave a bottle of white wine or champagne in the freezer to cool it quickly, and whether it matters. Or how long a bottle of red wine left overnight at $-5^{\circ} \mathrm{C}$ and needed for lunch should be left in the microwave oven and at what power?

Most of the work discussed was done in France, but maybe only laboratories there work on products of interest to a French audience, or perhaps they are better at publicity. It is both a weakness and a strength of this book that it is French. I cannot imagine anyone being funded to do this work in Britain or the United States, where there is less interest in food, but at least someone is doing it. Surely no one outside France cares whether the yolk is exactly in the middle of a boiled egg, and the English-speaking world, which is presumably the target for this edition, probably cares even less than the rest. In fact it is hard to see where the market for this book would be. Reading these intelligent but light-hearted columns in a monthly magazine is one thing, but buying and reading the book is quite another. Many of these stories date from 10 to 15 years ago, which is regarded as history by several of my colleagues, and even in food science the work is likely to have been overtaken by events.

The translation is generally very good, and the book makes a worthy attempt to explain the science to non-special ist readers. There are a few mistakes, but not important ones. For example, it refers to Brussels sprouts as a root vegetable - have I been eating the wrong bit all these years? In any case, the original sources are listed as 'further reading', although the individual chapters do not cite the references.

There is too much science to make this a mass-market book, and it is certainly not for the specialist food scientist. It would make a good present, perhaps, for a 'foodie' scientist, and I would put it on the reading list for an introductory food-science course, as it might interest some of the students.

John Piggott is in the Department of Bioscience, University of Strathclyde, Glasgow G1 1XW, UK.

\section{Correction}

The Science in Culture "Home from home"

(Nature 441,816; 2006) by Colin Martin should

have said that Benjamin Franklin died in

Philadelphia, rather than at Passy near Paris. 